\title{
BTrCP controls the lysosome-mediated degradation of CDK1, whose accumulation correlates with tumor malignancy
}

\author{
Joaquín Herrero-Ruiz ${ }^{1}$, Mar Mora-Santos ${ }^{1}$, Servando Giráldez ${ }^{1}$, Carmen Sáez ${ }^{2}$, \\ Miguel Á. Japón' ${ }^{2}$, Maria Tortolero ${ }^{1}$ and Francisco Romero ${ }^{1}$ \\ ${ }^{1}$ Departamento de Microbiología, Facultad de Biología, Universidad de Sevilla, Sevilla, Spain \\ 2 Instituto de Biomedicina de Sevilla (IBIS), Hospital Universitario Virgen del Rocío/CSIC/Universidad de Sevilla and \\ Departamento de Anatomía Patológica, Hospital Universitario Virgen del Rocío, Sevilla, Spain
}

Correspondence to: Francisco Romero, email: frport@us.es

Keywords: apoptosis/ autophagy/ BTrCP/ cancer/ CDK1

Received: May 22, $2014 \quad$ Accepted: July 26, $2014 \quad$ Published: July 27, 2014

This is an open-access article distributed under the terms of the Creative Commons Attribution License, which permits unrestricted use, distribution, and reproduction in any medium, provided the original author and source are credited.

\section{ABSTRACT}

In mammals, cell cycle progression is controlled by cyclin-dependent kinases, among which CDK1 plays important roles in the regulation of the G2/M transition, G1 progression and G1/S transition. CDK1 is highly regulated by its association to cyclins, phosphorylation and dephosphorylation, changes in subcellular localization, and by direct binding of CDK inhibitor proteins. CDK1 steady-state protein levels are held constant throughout the cell cycle by a coordinated regulation of protein synthesis and degradation. We show that CDK1 is ubiquitinated by the E3 ubiquitin ligase SCF ${ }^{\beta T r C P}$ and degraded by the lysosome. Furthermore, we found that DNA damage not only triggers the stabilization of inhibitory phosphorylation sites on CDK1 and repression of $C D K 1$ gene expression, but also regulates $\beta$ TrCP-induced CDK1 degradation in a cell type-dependent manner. Specifically, treatment with the chemotherapeutic agent doxorubicin in certain cell lines provokes CDK1 degradation and induces apoptosis, whereas in others it inhibits destruction of the protein. These observations raise the possibility that different tumor types, depending on their pathogenic spectrum mutations, may display different sensitivity to $\beta$ TrCP-induced CDK1 degradation after DNA damage. Finally, we found that CDK1 accumulation in patients' tumors shows a negative correlation with $\beta$ TrCP and a positive correlation with the degree of tumor malignancy.

\section{INTRODUCTION}

In mammalian cells, cell cycle progression is regulated by a series of cyclin-dependent kinases (CDKs), among which CDK1 has been primarily implicated in G2/M transition, mainly in association with cyclin B (see [1] for review). However, CDK1 is also able to regulate G1 progress and G1/S transition [2, 3]. Given its essential role in the cell cycle, CDK1 is highly regulated. First, $\mathrm{CDK} 1$ requires the association of one cyclin to recognize and phosphorylate its substrates [4]. Second, CDK1 is also regulated by phosphorylation and dephosphorylation and changes in subcellular localization $[5,6]$. Phosphorylation at Thr161 increases the complex activity, whereas phosphorylation at Thr14 and Tyr15 leads to inhibition of CDK1. Activating T-loop motif phosphorylation is carried out by CDK-activating kinases, whereas inhibitory kinases WEE1 and MYT1 phosphorylate Thr14 and Tyr15; the CDC25 phosphatase family members counteract this activity [7-9]. Finally, CDK1-cyclin complexes are also governed by direct binding of CDK inhibitor proteins [10]. On the other hand, CDK1 protein levels are held at a constant steady-state level throughout the cell cycle, which is maintained by a coordinated regulation of protein synthesis and degradation [11]. In fact, from G1/S transition to $\mathrm{G} 2 / \mathrm{M}$ boundary an active $C D K 1$ translation occurs accompanied by a concurrent degradation. However, little is known regarding the destruction of CDK1. It has been shown that CDK1 is downregulated under genotoxic stresses, that double-stranded RNAactivated protein kinase (PKR) is involved in the process and that PKR-mediated Tyr4-phosphorylation facilitates 


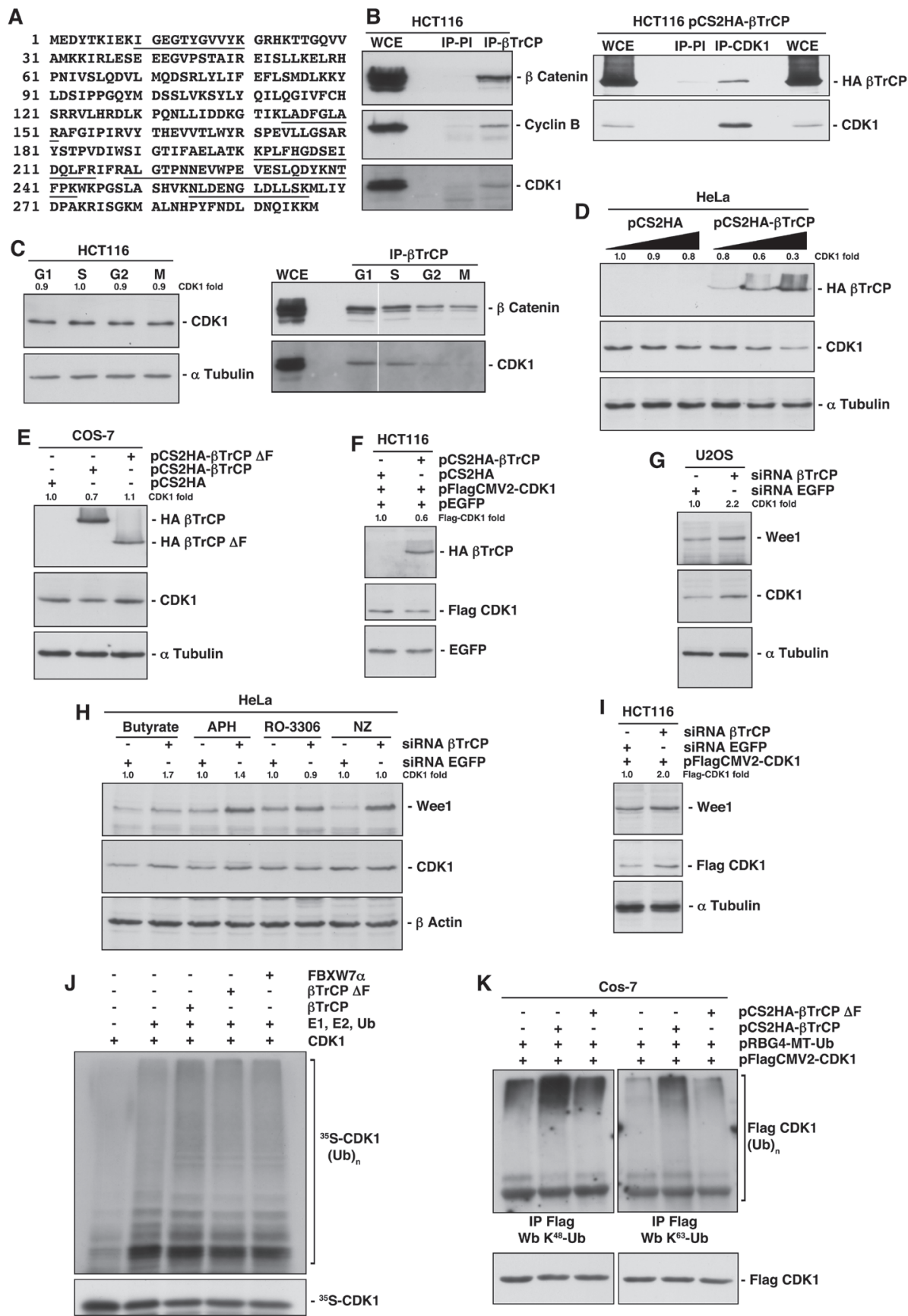

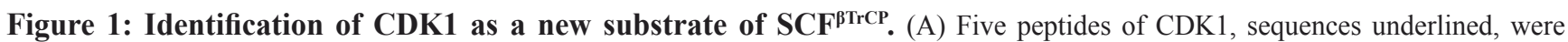
observed by MS/MS. (B) Whole cell extracts (WCE) from HCT116 or HCT116 transfected cells were used to immunoprecipitate $\beta$ TrCP (left panel) or CDK1 (right panel), and complexes were analyzed by immunoblotting. IP-PI: immunoprecipitation with normal rabbit (left panel) or mouse (right panel) sera. (C) HCT116 cells were synchronized in the different phases of the cell cycle as described in Methods, and analyzed by Western blot (left panel) or by immunoprecipitation (right panel). (D, E, F) HeLa, Cos-7 or HCT116 cells were transfected with the indicated plasmids and analyzed by immunoblot. (G) U2OS cells were interfered with the indicated siRNAs and analyzed by Western blot. (H) HeLa cells were interfered with the indicated siRNAs, and 24 hours before harvesting were synchronized using different drugs: butyrate (G1 phase), aphidicolin (APH, S phase), RO-3306 (G2 phase) or nocodazole (NZ, M phase). Extracts were blotted with the indicated antibodies. (I) HCT116 cells were transfected with pFlagCMV2-CDK1 and interfered with the indicated siRNAs, and analyzed by Western blot. (J) In vitro ubiquitin ligation assay of ${ }^{35} \mathrm{~S}$ labeled in vitro-transcribed/translated CDK1 was conducted in the presence or absence of the following products: cold in vitro-transcribed/translated $\beta \operatorname{TrCP}, \beta \operatorname{TrCP} \Delta \mathrm{F}$ or $\mathrm{FBXW} 7 \alpha, \mathrm{E} 1$ (His ${ }_{6}$-ubiquitin activating enzyme), E2 ( $\mathrm{His}_{6}-\mathrm{UbcH} 3$ and $\mathrm{UbcH} 5 \mathrm{a}$ ) and $\mathrm{Ub}$ (ubiquitin). Samples were incubated at $30^{\circ} \mathrm{C}$ for $1 \mathrm{~h}$. The bracket on the right side marks a ladder of bands corresponding to poly-ubiquitinated CDK1. (K) Cos-7 cells were transfected with the indicated plasmids and poly-ubiquitinated Flag-CDK1 visualized after Western blot of Flag immunoprecipitations. Anti-K ${ }^{48}-\mathrm{Ub}$ and $\mathrm{K}^{63}$-Ub polyclonal antibodies recognize K48- and K63-linkage specific ubiquitination, respectively. The quantitative fold change in CDK1 or Flag-CDK1 was determined relative to the loading control. 
CDK1 ubiquitination and proteosomal degradation [12]. Nevertheless, the CDK1-specific E3 ubiquitin ligase remains to be identified. In this study we found that CDK1 is ubiquitinated by $\mathrm{SCF}^{\beta \mathrm{TrCP}}$ and degraded by the lysosome. Furthermore, we analyzed the effect of DNA damage on CDK1 stability and on induction of apoptosis. Finally, we found that CDK1 accumulation in patients' tumors shows a negative correlation with $\beta \operatorname{TrCP}$ levels and a positive correlation with the degree of tumor malignancy.

\section{RESULTS}

\section{$\beta T r C P$ binds to CDK1}

To find new $\mathrm{SCF}^{\beta \mathrm{TrCP}}$ substrates, we searched for novel $\beta$ TrCP-interacting proteins. To address this, we performed anti-HA immunoprecipitation experiments of nuclear and cytosolic fractions from HA- $\beta$ TrCP transfected Cos-7 cells, followed by tandem mass spectrometry (MS/ MS). Immunoprecipitation reactions using normal mouse IgG served as negative controls. We detected CDK1 in cytosolic immunoprecipitates with $23.9 \%$ sequence coverage. Specifically, five different tryptic peptides (Fig 1A) were observed via MS/MS. To further validate the authenticity of this result, we confirmed the presence of CDK1 within the endogenous $\beta$ TrCP immunocomplex using Western blotting analysis (Fig 1B). Moreover, we also found cyclin B in the immunoprecipitation, which was also identified in the proteomic analysis with a single peptide. Anti- $\beta$-catenin was used as an internal control since commercial anti- $\beta \mathrm{TrCP}$ is not available for Western blot. In addition, we performed reciprocal immunoprecipitations using anti-CDK1 monoclonal antibodies and, as shown in figure $1 \mathrm{~B}, \mathrm{CDK} 1$ was also able to precipitate HA- $\beta \operatorname{TrCP}$. Furthermore, we studied at which phase the interaction with $\beta \operatorname{TrCP}$ occurs, as CDK1 protein is present in all phases of the cell cycle. Figure $1 \mathrm{C}$ shows that $\beta$ TrCP binds to CDK1 in $\mathrm{G} 1$ and $\mathrm{S}$ phases of the cell cycle.

\section{Expression of $\beta$ TrCP regulates CDK1 stability}

Next, we analyzed the effect of $\beta$ TrCP over- and underexpression on CDK1 levels. We found that increasing amounts of pCS2HA- $\beta \mathrm{TrCP}$ progressively reduced levels of endogenous CDK1 (Fig 1D). Furthermore, a $\beta \operatorname{TrCP}$ mutant lacking the F-box domain $(\beta \operatorname{TrCP} \Delta \mathrm{F})$ suppressed the CDK1 diminution (Fig 1E), involving full ubiquitin ligase in this scenario. Similar results were obtained when we explored this effect on a constitutively expressed CDK1, avoiding the potential transcriptional consequences of the $\beta \mathrm{TrCP}$ overexpression (Fig 1F). Reciprocal experiments were carried out using knockdown experiments. The efficiency of siRNA- $\beta \operatorname{TrCP}$ was verified interfering pCS2HA- $\beta$ TrCP transfected HeLa cells by testing HA- $\beta$ TrCP depletion (supplementary Fig S1A). We then interfered several cell lines and observed an increase of CDK1 upon $\beta \mathrm{TrCP}$ depletion, similar to that achieved for WEE1, a known substrate of $\operatorname{SCF}^{\beta \operatorname{TrCP}}$ (Figs 1G-1I), and a longer half-life of the protein (supplementary Fig S1B). This increase was only perceived in $\mathrm{G} 1$ and $\mathrm{S}$ phases (Fig $1 \mathrm{H})$, precisely where the $\beta \mathrm{TrCP} / \mathrm{CDK} 1$ interaction is detected. Likewise, $\beta \mathrm{TrCP}$ depletion also augmented transfected Flag-CDK1 (Fig 1I and supplementary Fig $\mathrm{S} 1 \mathrm{C})$, ratifying the post-translational effect of $\beta \mathrm{TrCP}$
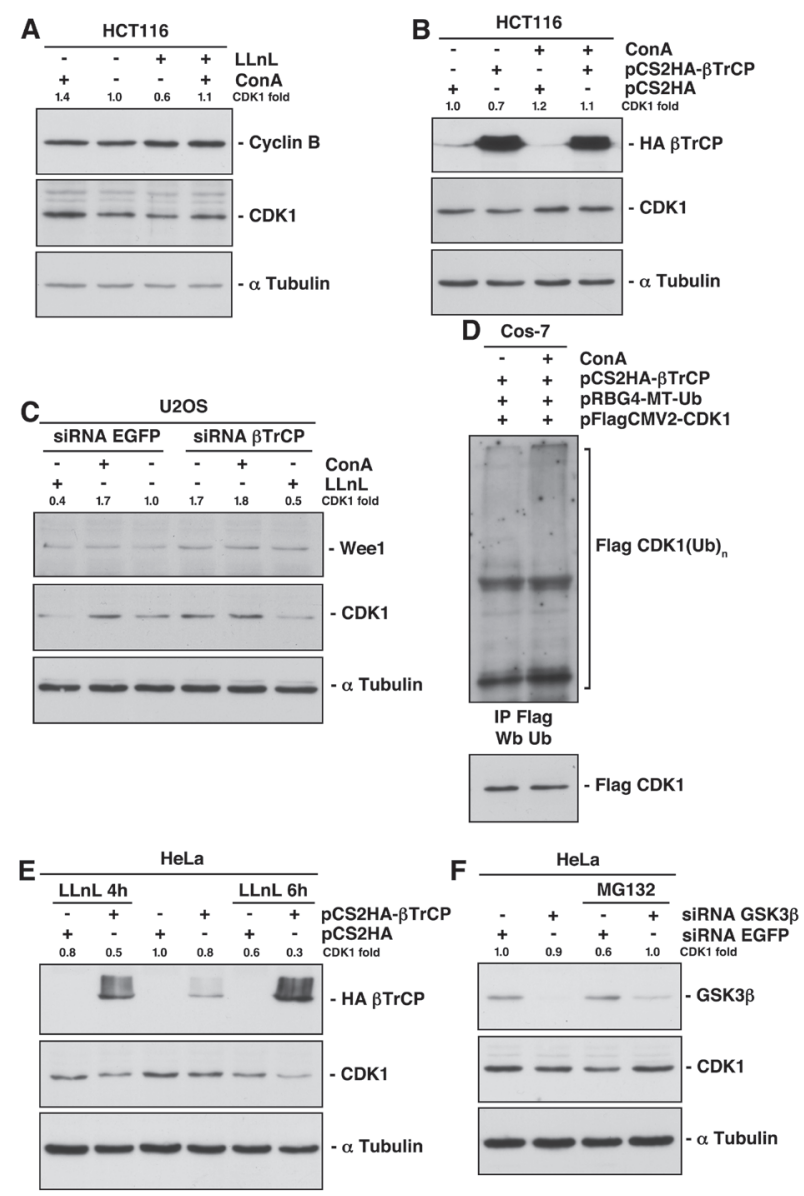

Figure 2: Lysosomal-mediated CDK1 degradation involves both $\mathrm{SCF}^{\beta \mathrm{TrCP}}$-dependent and independent mechanisms. (A) HCT116 cells were treated with proteasomal (LLnL) or/and lysosomal (ConA) inhibitors for 4 hours and protein expression analyzed by immunoblot. (B) HCT116 cells were transfected with the indicated plasmids and treated or not with ConA 4 hours before harvesting. Total extracts were blotted with different antibodies. (C) U2OS cells were interfered and treated with LLnL or ConA 4 hours before harvested. Extracts were analyzed by immunoblot. (D) Comparison of CDK1 ubiquitination from transfected cells treated or not with ConA. Flag-CDK1 was immunoprecipitated and blotted with the indicated antibodies. (E, F) HeLa cells were transfected or interfered and treated with proteasome inhibitors. Extracts were analyzed by Western blot. The quantitative fold change in CDK1 was determined relative to the loading control. 
expression on CDK1. Together, these results reveal that $\beta \operatorname{TrCP}$ regulates CDK1 stability.

\section{$\mathrm{SCF}^{\beta \mathrm{TrCP}}$ ubiquitinates CDK1 both in vitro and in vivo}

To ascertain that $\mathrm{SCF}^{\beta \mathrm{TTCP}}$ promotes CDK1 ubiquitination, we carried out both in vitro and in vivo assays. First, we examined the in vitro ubiquitination of transcribed and translated ${ }^{35} \mathrm{~S}$-labeled CDK1 by SCF ${ }^{\beta T r C P}$ (Fig 1J). In the presence of ubiquitin, high molecular weight ubiquitinated derivatives of CDK1 were formed (third lane), while $\beta \operatorname{TrCP} \Delta \mathrm{F}$ prevented ubiquitination. $\mathrm{SCF}^{\mathrm{FBXW7a}}$ was used as a selectivity control of CDK1ubiquitin ligation for $\mathrm{SCF}^{\mathrm{BTC} C \mathrm{P}}$. These data indicate that $\mathrm{SCF}^{\beta \mathrm{TrCP}}$ directly ubiquitinates $\mathrm{CDK} 1$, at least, in vitro. Next, we immunoprecipitated Flag-CDK1 in the presence of ubiquitin and $\beta \operatorname{TrCP}$ (or $\beta \operatorname{TrCP} \Delta \mathrm{F}$ ). We detected ubiquitinated forms of CDK1 with an antiubiquitin K48 specific antibody, which recognizes K48linked polyubiquitin chains as mediators of proteasomal degradation, or an anti-ubiquitin K63 specific antibody, which binds to K63-linked polyubiquitin chains, that act in a range of other processes [13] (Fig 1K). In both cases, CDK1 was ubiquitinated by $\mathrm{SCF}^{\beta \mathrm{TrCP}}$.

\section{Ubiquitin-mediated lysosomal degradation of CDK1}

Eukaryotic cells utilize two major routes to effectively target a wide range of proteins for degradation: the ubiquitin/proteasome system and the autophagy/ lysosome pathway [14]. To determine whether one or both of these systems were involved in the $\beta \operatorname{TrCP}$ regulation of CDK1 stability, we tested the effect of LLnL and concanamycin A (ConA) on CDK1 stability and their influence when cells were transfected or depleted of $\beta \operatorname{TrCP}$. We found that ConA, a specific lysosomal inhibitor, increased the amount of CDK1. Unexpectedly, the proteasomal inhibitor LLnL, not only did not increase, but decreased CDK1 below control levels (Fig 2A). In fact, CDK1 was internalized to discrete autophagic vesicles within the cytoplasm after LLnL treatment (supplementary Fig S2A). Moreover, ConA partially prevented this reduction (Fig 2A, fourth lane), indicating that treatment with LLnL induced lysosomal degradation of CDK1. As a control, we analyzed the behavior of cyclin $\mathrm{B}$, which was not affected by ConA and increased with LLnL treatment. The proteasomal inhibitors' effect on CDK1 was confirmed in all cell lines tested and using two different inhibitors (supplementary Fig S2B). In addition, treatment with ConA substantially avoided the CDK1 degradation caused by $\beta \operatorname{TrCP}$ overexpression (Fig $2 \mathrm{~B}$ ). Furthermore, the increase of CDK1 induced by depletion of $\beta \operatorname{TrCP}$ was comparable to that caused by the treatment with ConA (Fig 2C, fourth vs. second lanes), and the latter could not enhance CDK1 levels in $\beta \operatorname{TrCP}$ interfered cells (Fig 2C, fourth vs. fifth lanes). These results strongly support the idea that $\beta \operatorname{TrCP}$ induces the degradation of CDK1 through the lysosome. This conclusion was validated by comparing the appearance of ubiquitinated forms of CDK1 promoted by $\beta \operatorname{TrCP}$ overexpression in the presence or absence of ConA (Fig 2D). Taken together, we have demonstrated that CDK1 ubiquitination induced by $\mathrm{SCF}^{\beta \mathrm{TrCP}}$ provokes its lysosomal degradation.

\section{GSK3 $\beta$ mediates CDK1 degradation induced by proteasome inhibition}

We previously described how inhibition of the proteasome by LLnL treatment decreased CDK1 levels (Fig 2A). The degradation of CDK1 induced by LLnL could not be reversed by $\beta \operatorname{TrCP}$ depletion (Fig $2 \mathrm{C}$, first vs. sixth lanes). Similarly, the presence of LLnL in cells overexpressing $\beta \mathrm{TrCP}$ not only does not reduce the CDK1 degradation, but further increases it (Fig 2E). This is because $\mathrm{SCF}^{\beta \mathrm{TrCP}}$ is not involved in the proteasomal mediated degradation of CDK1, and because LLnL protects $\beta \operatorname{TrCP}$ itself from degradation by the proteasome (compare the HA- $\beta \operatorname{TrCP}$ expression of the fourth lane with that of second and sixth lanes of Fig 2E).

A recent report provides evidence supporting a role of GSK $3 \beta$ in the regulation of autophagy activation in response to proteasome inhibition [15]. To investigate whether GSK $3 \beta$ mediates CDK1 degradation after LLnL treatment, we interfered HeLa cells and found that GSK3 $\beta$ depletion impedes the lysosomal destruction of CDK1 (Fig $2 \mathrm{~F})$. Therefore, these results reveal that GSK $3 \beta$ signaling determines a lysosomal $\beta \mathrm{TrCP}$-independent degradation of CDK1 induced by proteasome inhibition.

\section{Identification of a $\beta$ TrCP-binding site in CDK1}

Sequence analysis of CDK1 revealed the sequence EEGVPS, at residues 41-46, which is evolutionary conserved (Fig 3A). This sequence is very similar to the phosphodegron motif (DSGXXS) recognized by $\beta \mathrm{TrCP}$, especially considering that phosphoserine may be replaced by an acidic amino acid (glutamic or aspartic acids) [16]. We generated a CDK1 mutant in its $\beta$ TrCP-binding motif (CDK1- $\beta M$ ) by mutating residues 41,42 and 46 to Ala or Gly. In addition, we mutated Thr47, next to the phosphodegron, since the consensus motif sometimes has from two to four amino acids of any type $\left(\mathrm{X}_{2-4}\right)$ [17]. To verify that the CDK1- $\beta \mathrm{M}$ was insensitive to $\beta \operatorname{TrCP}$ mediated degradation, we overexpressed both $\beta \operatorname{TrCP}$ and $\beta \operatorname{TrCP} \Delta \mathrm{F}$ proving that neither degraded CDK1- $\beta \mathrm{M}$ (Fig $3 \mathrm{~B}) . \beta \operatorname{TrCP}$ was unable to associate with (Fig $3 \mathrm{C}$ ) nor ubiquitinate in vivo to the mutated version of CDK1 (Fig 3D). Given these data, we have identified the binding site 


\section{of $\beta \operatorname{TrCP}$ to $\mathrm{CDK} 1$.}

\section{DNA damage regulates $\beta$ TrCP-induced CDK1 degradation in a cell type-dependent manner}

Since a previous study reported that CDK1 was downregulated upon genotoxic stresses [12], we studied the role of $\beta \operatorname{TrCP}$ on CDK1 stability after DNA damage. To this end, several cells lines were treated with doxorubicin for $24 \mathrm{~h}$ and extracts analyzed by Western blot. We found that CDK1 stability depended on the cell types: CDK1 decreased in HEK293T and LNCaP cells, and increased in HCT116, HeLa and PC3 cells (Fig 4A). This behavior does not depend on the accumulation of cells in a specific phase of the cell cycle because the CDK1 amount is constant throughout the cell cycle
[11]. In addition, doxorubicin treatment led to a decline in the G1:G2/M ratios in all cell lines, as expected (supplementary Fig S3) [18]. To eliminate the potential transcriptional effect of doxorubicin, before treating cells, protein synthesis was blocked with cycloheximide. Figure 4B shows that CDK1 protein from HCT116 cells was more stable when cells were treated with doxorubicin than when left untreated. Similar results were obtained for p53. Therefore, in HCT116 cells, DNA damage protects CDK1 from degradation. To inquire into mechanisms underlying CDK1 stability after DNA damage, we studied whether PKR was involved in CDK1 regulation, as previously suggested. Treatment with $\mathrm{C} 16$, a PKR inhibitor, or a specific PKR siRNA, both prevented the increase of CDK1 and even caused a reduction of CDK1 levels, after doxorubicin treatment (Figs 4C and 4D). Moreover, activation of PKR with poly(I:C) induced an

\section{A}

\begin{tabular}{|c|c|}
\hline $\begin{array}{l}\beta T \text { TrCP CONSENSUS } \\
\text { CDK1 }\end{array}$ & $\operatorname{DSGX}_{2-4} \mathrm{~S}$ \\
\hline 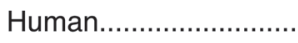 & ...EEGVPST \\
\hline Mouse... & ..EEGVPST \\
\hline Rat.. & ...EEGVPST \\
\hline D. rerio. & ...EEGVPST \\
\hline X. laevis.. & ...EEGVPST \\
\hline C. elegans... & ..DEGVPST \\
\hline D. melanogaster....... & ...DEGVPST \\
\hline 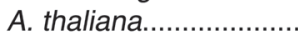 & ...DEGVPST \\
\hline urelia.............. & ...DEGVPST \\
\hline visiae.............. & ...DEGVPST \\
\hline ho & ...SEGVPS \\
\hline
\end{tabular}

CDK1 WT.................. EEGVPST
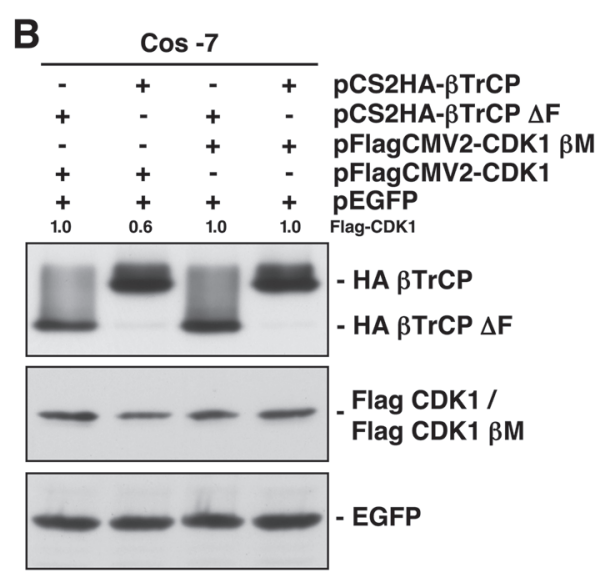

D

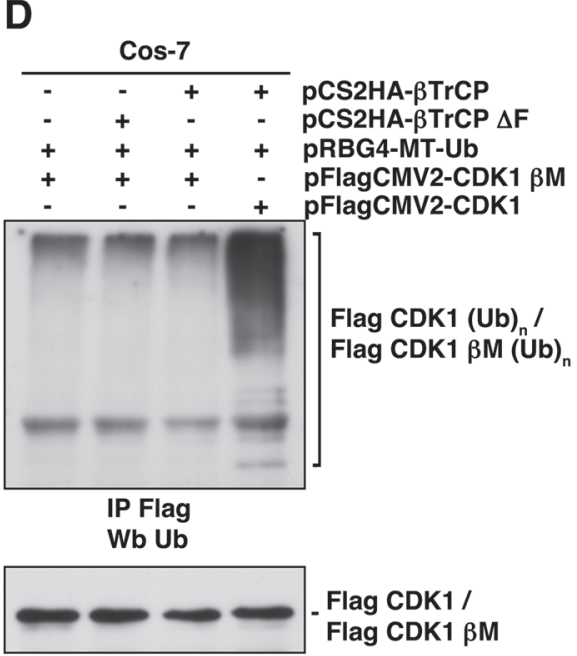

Figure 3: Identification of the $\beta \operatorname{TrCP}$ recognition motif in CDK1, whose mutation avoids both $\beta \operatorname{TrCP} / \mathrm{CDK} 1$ interaction, and CDK1 ubiquitination and degradation. (A) Alignment of the putative $\beta \operatorname{TrCP}$ consensus sequence identified in human $C D K 1$ (residues 41 to 46) with other orthologs. Arrows indicate the amino acid changes performed to obtain a CDK1 mutated in this motif (CDK1 $\beta \mathrm{M}$, CDK1 $\beta \operatorname{TrCP}$ mutant). (B) Cos-7 cells were transfected with the indicated plasmids and extracts analyzed by immunoblot. (C) $\beta \operatorname{TrCP}$ was immunoprecipitated from Cos-7 transfected cells, and coimmunoprecipitation of Flag-CDK1 or Flag-CDK1 $\beta M$ studied with anti-Flag. Anti- $\beta$ catenin was used as a control of $\beta \mathrm{TrCP}$ immunoprecipitation. IP-PI: immunoprecipitation with normal rabbit serum. WCE: whole cell extracts. (D) Cos-7 cells were transfected with the indicated plasmids, and Flag-CDK1 or Flag-CDK1 $\beta$ M ubiquitination obtained after Flag immunoprecipitation and Western blot using anti-ubiquitin. The quantitative fold change in Flag-CDK1 was determined relative to the loading control. 


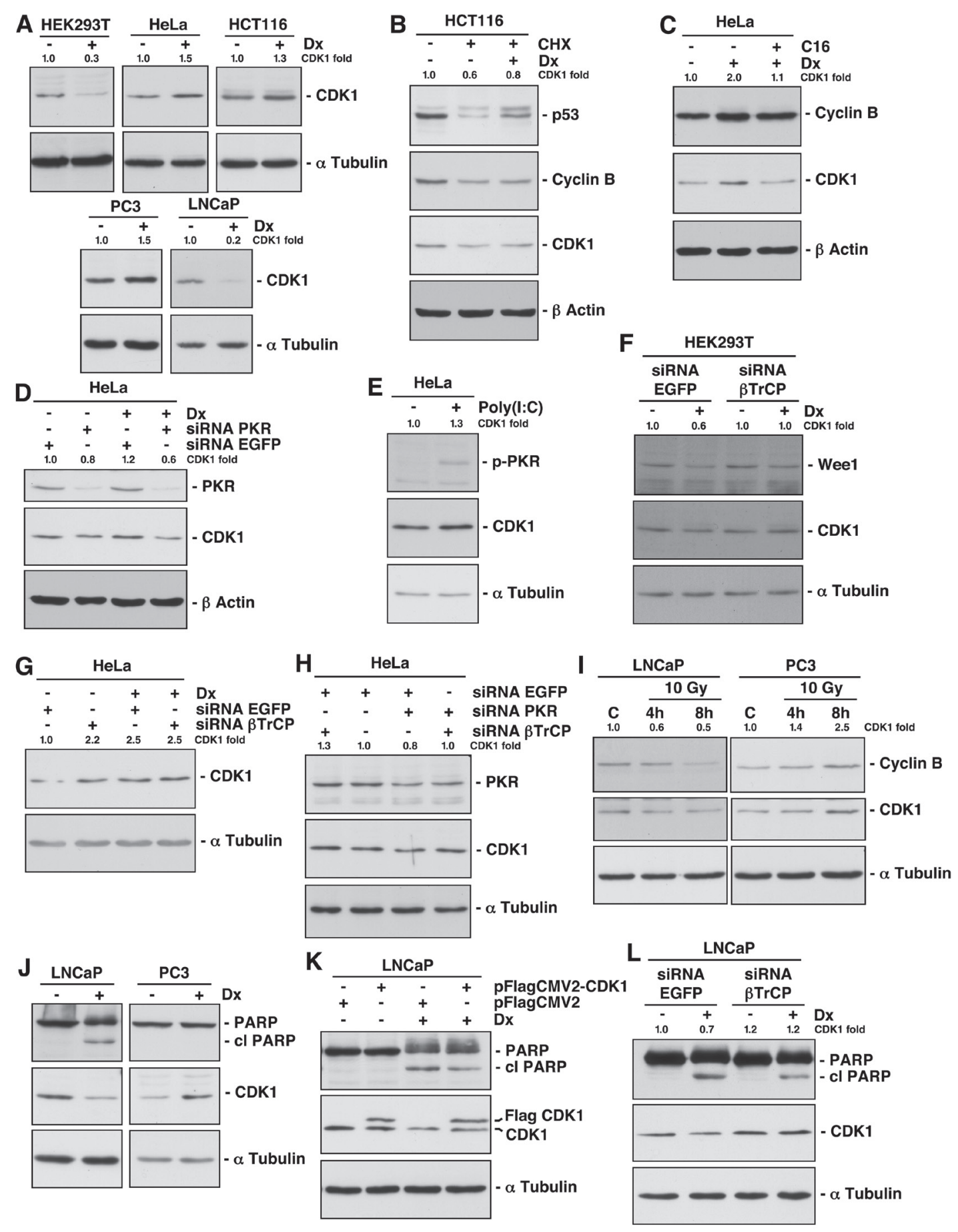

Figure 4: DNA damage alters $\beta$ TrCP-induced CDK1 degradation and its corresponding induction of apoptosis in a cell type dependent manner. (A) Cell lines were treated with doxorubicin (Dx) for 24 hours, and CDK1 expression analyzed by immunoblot. (B) Protein synthesis of HCT116 cells was arrested with cycloheximide (CHX) and treated or not with Dx for 10 hours. Extracts were analyzed by Western blot. (C) HeLa cells were treated with Dx for 24 hours and with C16, a PKR inhibitor, 2 hours before harvested. Proteins expression was studied with different antibodies. (D) HeLa cells were interfered with the indicated siRNAs, and 24 hours before harvested, treated with Dx. Extracts were blotted with the indicated antibodies. (E) HeLa cells were treated with poly(I:C), a PKR activator, for 6 hours, and extracts were analyzed by immunoblot. p-PKR indicates phospho-PKR (Thr451). (F, G) HEK293T and HeLa cells were treated as in (D). (H) HeLa cells were interfered with the indicated siRNAs and extracts analyzed by immunoblot. (I) $\mathrm{LNCaP}$ and PC 3 cells were $\gamma$-irradiated to a dose of $10 \mathrm{~Gy}$ and harvested $4 \mathrm{~h}$ and $8 \mathrm{~h}$ later. Extracts were blotted with the indicated antibodies. C: control. (J) LNCaP and PC3 cells were treated as in (A). cl PARP: cleaved PARP. (K) LNCaP cells were transfected with the indicated plasmids and treated or not with Dx for 24 hours before harvested. Extracts were analyzed by Western blot. cl PARP: cleaved PARP. (L) LNCaP cells were treated as in (D). cl PARP: cleaved PARP. The quantitative fold change in CDK1 was determined relative to the loading control. 
increase of CDK1 (Fig 4E). In conclusion, the doxorubicin effect on CDK1 is mediated by PKR, both in cells where CDK1 increases (Fig 4) as in cells where decreases (supplementary Fig S4A, and [12]).

We next employed HeLa cells as a model in which CDK1 increases after doxorubicin treatment, and HEK293T, in which CDK1 decreases in order to study whether $\beta$ TrCP was implicated in CDK1 stability after DNA damage. Knockdown experiments showed that $\beta$ TrCP avoided the CDK1 diminution after doxorubicin in HEK293T cells (Fig 4F). However, the siRNA $\beta$ TrCP-induced increase of CDK1 in HeLa cells was not augmented by treatment with the drug, suggesting that the effect of doxorubicin is produced through the loss of function of $\beta \operatorname{TrCP}$ (Fig 4G). This hypothesis was confirmed by the fact that the reduction of CDK1 obtained by siRNA PKR in HeLa cells was prevented in the absence of $\beta \operatorname{TrCP}($ Fig $4 \mathrm{H})$. Taken together, we can conclude that $\mathrm{SCF}^{\beta \mathrm{TrCP}}$ mediates CDK1 stability after DNA damage.

\section{Implication of CDK1 degradation in the induction of apoptosis after DNA-damage}

As we have demonstrated above, CDK1 is degraded in $\mathrm{LNCaP}$ and increased in PC3 cells after doxorubicin treatment. We were interested to study whether gamma irradiation induced similar results, because it is also used for the treatment of a wide variety of cancers, with probably less side effects. Figure 4I shows that both cell lines behave in the same way following irradiation as after doxorubicin treatment. A number of studies have established a correlation between CDK1 activity and apoptosis induced by DNA damage [19]. In fact, the CDK1 inhibitor RO-3306 induced apoptosis in all cell lines tested (supplementary Fig S4B). Thus, we analyzed whether CDK1 degradation was related to the onset of apoptosis. For this, we examined the appearance of the $85 \mathrm{kDa}$-cleaved fragment of PARP protein as a hallmark of apoptosis. We found that doxorubicin treatment induced PARP cleavage and degradation of CDK1 in LNCaP but not in PC3 cells, suggesting that both events could be associated (Fig 4J). To validate this result, we overexpressed CDK1 in LNCaP cells and found a significant PARP cleavage reduction after doxorubicin (Fig
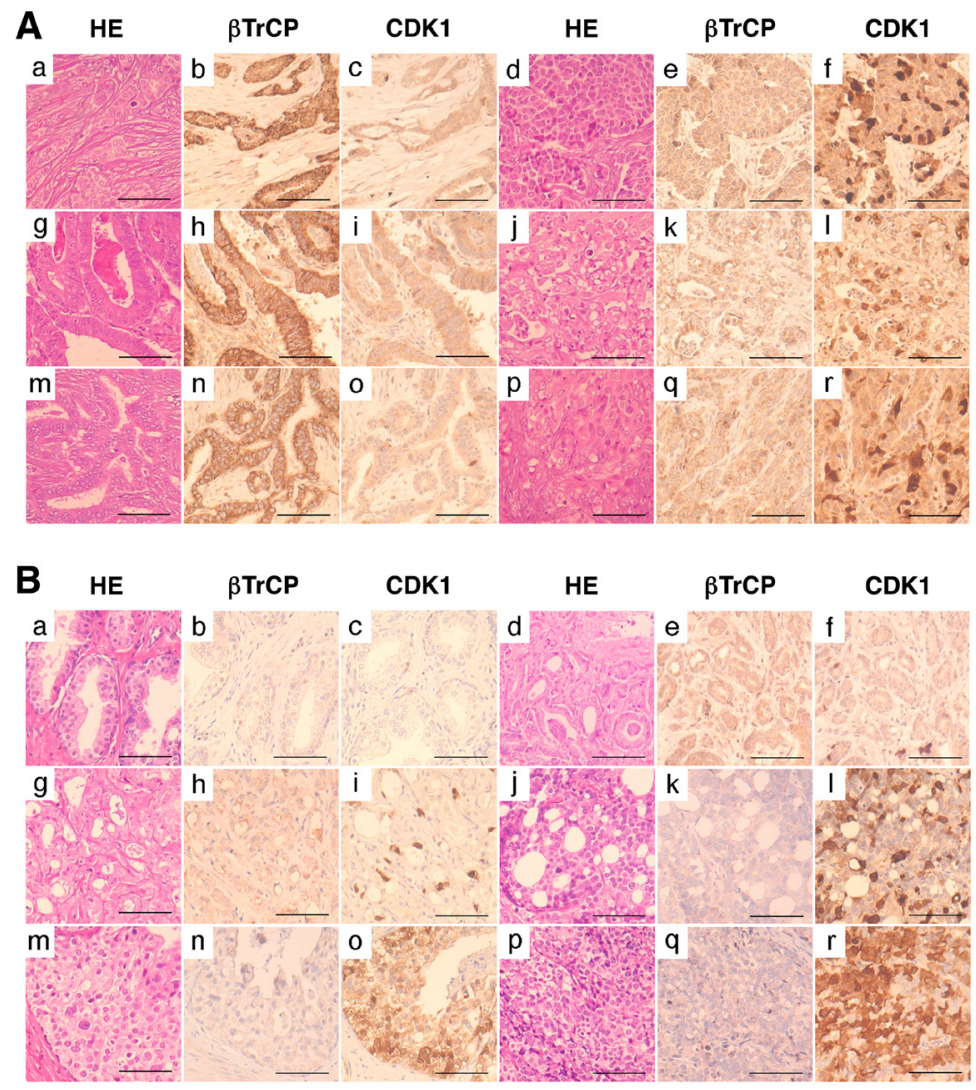

Figure 5: CDK1 accumulation in human tumors shows inverse correlation with $\beta$ TrCP expression and is associated with tumor grade. (A) Immunohistochemical expression of $\beta \mathrm{TrCP}$ and $\mathrm{CDK} 1$ in human breast carcinomas (a-f), gastric carcinomas

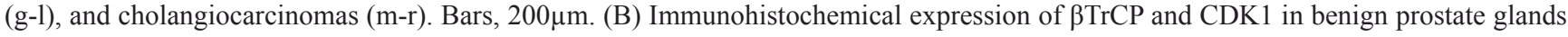
(a-c), low grade prostatic carcinomas (d-i), and high grade prostatic carcinomas (j-r). HE, hematoxylin-eosin. Bars, $200 \mu \mathrm{m}$. 
4K). Similar results were observed using HEK293T cells (supplementary Fig S4C). Furthermore, $\beta$ TrCP depletion in doxorubicin treated LNCaP cells, not only avoided CDK1 degradation as expected, but also diminished PARP cleavage (Fig 4L). Hence, we can deduce that $\beta$ TrCPmediated CDK1 degradation intervenes in apoptosis induced by DNA-damage.

\section{CDK1 accumulation in human tumors shows inverse correlation with $\beta \operatorname{TrCP}$ expression}

According to our results, CDK1 expression in tumors could be modulated by $\beta \operatorname{TrCP}$ expression and have an impact on the degree of malignancy of tumors. To check this, we first analyzed the immunohistochemical expression of $\mathrm{CDK} 1$ and $\beta \mathrm{TrCP}$ in a tissue microarray containing 66 human carcinomas of different sites. Forty three (65.1\%) carcinomas showed high levels of CDK1. Of these 43 tumors, 40 showed reduced (low to moderate) levels of $\beta \mathrm{TrCP}$, while 13 out of 23 tumors with low CDK1 showed high $\beta$ TrCP expression. On the other hand, 50 (75.7\%) carcinomas showed reduced levels of $\beta$ TrCP. Of these 50 tumors, 40 expressed high CDK1 levels, and conversely, CDK1 expression was reduced in 13 out of 16 carcinomas with high $\beta$ TrCP. Representative immunostains are shown in Figure 5A. Inverse correlation between $\mathrm{CDK} 1$ and $\beta \mathrm{TrCP}$ expression was statistically significant in this series of tumors $(\mathrm{p}<0.05$, from Fisher's exact test). The fact that high CDK1, low $\beta \operatorname{TrCP}$ phenotype was seen in a majority of tumors may reflect the proportion of high grade carcinomas in this tissue microarray. To further address this, we analyzed the accumulation of CDK1 in 109 carcinomas of the prostate (60 low grade, 49 high grade) and looked for its association with tumor grade. Fifty eight (96.7\%) low grade carcinomas showed low levels of CDK1, while $39(79.6 \%)$ high grade carcinomas expressed elevated levels of CDK1. Representative immunostains are shown in Figure 5B. Association of CDK1 accumulation and tumor grade was statistically significant in this series of prostatic cancers $(\mathrm{p}<0.05$, from Fisher's exact test). These results suggest that loss of $\beta \operatorname{TrCP}$ may facilitate $\mathrm{CDK} 1$ accumulation in tumors, particularly in those of high grade of malignancy.

\section{DISCUSSION}

It has been shown that CDK1 transcription/ translation oscillates in a cell cycle-specific manner [11]. However, CDK1 levels are constant during the normal cell cycle. Therefore, degradation of the protein must be strictly regulated. We have demonstrated that $\mathrm{SCF}^{\beta \mathrm{TrCP}}$ contributes to maintain invariable the CDK1 level throughout the cell cycle by its lysosome-mediated degradation. Moreover, proteasome inhibition further induced the lysosomal degradation of CDK1, in which GSK3 $\beta$ signaling was involved. However, recently Yoon et al. reported that CDK1 increased after MG132 treatment [12]. The reasons for this discrepancy are unclear, but we confirmed CDK1 degradation after proteasome inhibition in the seven cell lines tested.

DNA damage induces cell cycle arrest through several mechanisms [20]. In regard to CDK1, damage triggers stabilization of inhibitory phosphorylation sites on CDK1 [21] and repression of CDK1 gene expression [22]. Here, we present an additional novel mechanism that involves CDK1 polyubiquitination by $\mathrm{SCF}^{\mathrm{BrCP}}$ and its degradation by the lysosome. This effect appears to depend on the cell type, since in some cell lines DNA damage induces CDK1 degradation, whereas in others it prevents destruction of the protein, probably due to changes in the folding of CDK1. Although more studies are required to confirm this, the difference could reflect the distinct tumorigenic ability of the diverse cell lines. Thus, HEK cells have been reported to have a moderate tumorigenic potential, being used as a cellular model for normal human cells to study the oncogenic potential of a number of genes $[23,24]$. LNCaP cells are non-invasive and poorly tumorigenic, whereas PC3 cells are highly tumorigenic and invasive [25], and similarly HeLa and HCT116 are highly tumorigenic [26, 27]. Figure 6 shows

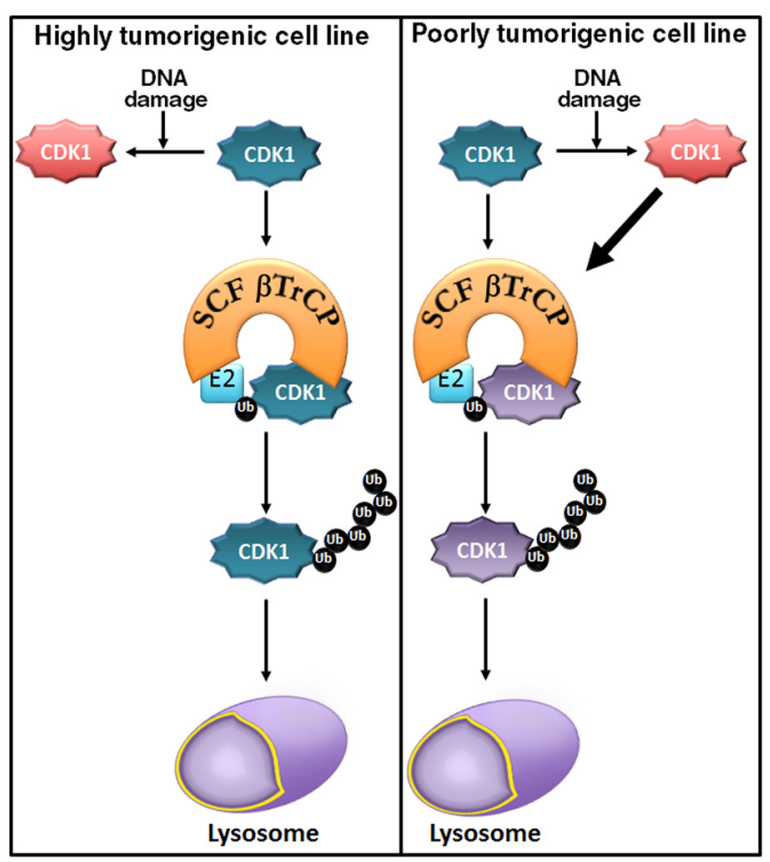

Figure 6: A model of the mechanism of CDK1 degradation. In all cell lines tested, CDK1 (in blue) is ubiquitinated and degraded by $\mathrm{SCF}^{\beta \operatorname{TrCP}} /$ lysosome in control conditions (basal phosphorylation status). In highly tumorigenic cell lines, DNA damage inhibits CDK1 degradation (in red), whereas in poorly tumorigenic cell lines it enhances CDK1 degradation (thick black arrow). CDK1 (in purple) indicates both CDK1 from control conditions and after DNA damage. Ub (in black): ubiquitinated CDK1. 
a model of the mechanism of CDK1 degradation in accord with our results.

It has been published that PKR phosphorylates amino-terminal Tyr4 of CDK1 inducing its ubiquitination in cells where CDK1 is degraded after doxorubicin treatment [12]. In fact, we have found that PKR modifies the stability of CDK1 mediated by $\mathrm{SCF}^{\beta \operatorname{TrCP}} /$ lysosome after DNA damage. We have identified the $\beta$ TrCP-binding site in CDK1 between residues 41 to 46 . How Tyr4 phosphorylation may alter the ubiquitination/degradation induced by $\beta \operatorname{TrCP}$ is a matter that remains to be elucidated. Perhaps, CDK1 is folded in such a way that its phosphorylation changes the $\beta \operatorname{TrCP}$ access to the molecule allowing or not its ubiquitination and degradation, as has been previously suggested [12].

Furthermore, in this study we reveal that $\beta \mathrm{TrCP}$ mediated CDK1 degradation is associated with genotoxinmediated apoptosis. Thus, doxorubicin-mediated apoptosis in LNCaP cells was attenuated by CDK1 overexpression or by $\beta \operatorname{TrCP}$ depletion. In concurrence with our results, it has been reported that PKR mediates the inhibition of cell proliferation and apoptosis under stress conditions in which CDK1 is degraded under such conditions $[12,28]$. More importantly, cells where CDK1 was not degraded after DNA-damage did not induce PARP cleavage and, consequently, did not progress into apoptosis. These data are of particularly high impact, given that by a simple analysis of biopsy samples from patients treated with genotoxins or gamma radiation, a read-out could be obtained as to whether tumors will suffer apoptosis or recurrence.

Finally, in different tumor types from patient samples, we showed an inverse correlation between CDK1 accumulation and $\beta \operatorname{TrCP}$ levels. We also observed a positive correlation between CDK1 accumulation and the degree of malignancy of tumors. $C D K 1$ gene amplification or overexpression occurs in human tumors, but our results also suggest that loss of $\beta \mathrm{TrCP}$ may facilitate $\mathrm{CDK} 1$ accumulation. These results support the development of molecules that promote the degradation of CDK1 as a future therapeutic target for cancer treatment.

\section{METHODS}

\section{Plasmids, cloning, point mutation and sequencing}

pCS2HA- $\beta \operatorname{TrCP}, \quad$ pCS2HA- $\beta \operatorname{TrCP} \Delta \mathrm{F}$ and empty vectors have been previously described [2931]. pEGFP-N1 and pRBG4-MT-Ub were from BD Biosciences and ATCC, respectively. pFlagCMV2-CDK1 and pCMVHA-FBXW7 $\alpha$ were obtained by cloning the corresponding PCR fragments in pFlagCMV2 and pCMVHA, respectively. Flag-CDK1 $\beta$ M was constructed using the "Transformer Site-Directed Mutagenesis Kit" from BD Biosciences. Construct sequences and point mutations were verified on both strands with an automatic sequencer.

\section{Cell culture, cell synchronization, transient transfections, drugs and $\gamma$-irradiation}

Routinely, HeLa, HCT116, Cos-7, U2OS, HEK293T, PC3 and LNCaP (from ATCC), and HCT116 p53 ${ }^{--}$cells [32] were grown in Dulbecco's modified Eagle's medium (Lonza) as described [33]. Cells enriched in the different phases of the cell cycle were also obtained as previously described [34], or by treating them with butyrate $(6 \mathrm{mM})$, aphidicolin (APH $1 \mu \mathrm{M})$, RO-3306 $(\mathrm{RO} 9 \mu \mathrm{M})$ or nocodazole $(\mathrm{NZ} 10 \mu \mathrm{M})$, all from Sigma, and confirmed by flow cytometry. DNA constructs were transiently transfected by electroporation or using lipid transfection reagents (Lipofectamine (Invitrogen) or Xfect (Clontech)), and $18 \mathrm{~h}$ or $48 \mathrm{~h}$ post-transfection, respectively, cells were harvested and lysed. For some experiments, cells were treated with Ac-LLnL-CHO (LLnL 100 $\mu \mathrm{M}$, Sigma), MG132 (MG 20 $\mathrm{M}$, Boston Chemical), concanamycin A (ConA 50nM, Sigma), doxorubicin (Dx $1 \mu \mathrm{M}$, Sigma), C16 (1 $\mathrm{M}$, Merck), poly(I:C) (500ng/ml, Sigma) or cycloheximide (CHX $50 \mu \mathrm{g} / \mathrm{ml}$, Sigma), and harvested at various times. Where indicated, cells were $\gamma$-irradiated to a dose of $10 \mathrm{~Gy}$ and harvested $4 \mathrm{~h}$ and $8 \mathrm{~h}$ later.

\section{Subcellular fractionation and lysis}

Subcellular fractionation was carried out as described [35]. Whole cell extracts were prepared at $4^{\circ} \mathrm{C}$ in $420 \mathrm{mM} \mathrm{NaCl}, 10 \mathrm{mM}$ Tris- $\mathrm{HCl}$ (pH 7.5), 1\% Nonidet P-40 (NP40), 10\% glycerol, 1mM PMSF (phenylmethylsulfonyl fluoride), $1 \mu \mathrm{g} / \mathrm{ml}$ aprotinin, $1 \mu \mathrm{g} / \mathrm{ml}$ pepstatin, $1 \mu \mathrm{g} / \mathrm{ml}$ leupeptin and $10 \mu \mathrm{g} / \mathrm{ml}$ chymostatin for $20 \mathrm{~min}$. Extracts were centrifuged at 20,000 $\mathrm{g}$ for $20 \mathrm{~min}$ and supernatants frozen in liquid nitrogen and stored at $-80^{\circ} \mathrm{C}$. Protein concentration was determined using the Bradford assay (Bio-Rad). After DNA damage, cell extracts were treated with lambda protein phosphatase ( $\lambda$-PP) [36].

\section{Small interfering RNA (siRNA) assays}

Cells were interfered with $\beta \operatorname{TrCP} 1 / 2-$, GSK3 $\beta$ - or PKR-siRNA [37-39] using the Oligofectamine method (Invitrogen) to suppress the expression of endogenous genes. EGFP-siRNA [38] was used as a non-specific control. Cells were harvested $48 \mathrm{~h}$ post-transfection and reduction of protein levels confirmed by Western blotting. 


\section{Electrophoresis, Western blot analysis and antibodies}

Proteins were separated by SDS-polyacrylamide gel electrophoresis (SDS-PAGE) and gels were electroblotted onto nitrocellulose membranes and probed with the following antibodies: anti-HA-peroxidase monoclonal antibody (Roche); anti-CDK1 and anti-p53 monoclonal antibodies (Santa Cruz); anti- $\alpha$-Tubulin, anti-Flag and anti- $\beta$-actin monoclonal antibodies (Sigma); anti-GFP polyclonal antibody (Immune Systems Ltd.); anti-WEE1 and anti-K48 and anti-K63-linkage specific polyubiquitin polyclonal, and anti-ubiquitin monoclonal antibodies from Cell Signaling; anti-phosphoPKR monoclonal antibody (Epitomics); and anti-cyclin $\mathrm{B}$, anti- $\beta$-catenin, antiGSK3 $\beta$, anti-PARP and anti-PKR monoclonal antibodies (BD Biosciences). Peroxidase-coupled donkey anti-rabbit IgG and sheep anti-mouse $\operatorname{IgG}$ were obtained from GE Healthcare. Immunoreactive bands were visualized using the Enhanced Chemiluminescence Western blotting system (ECL, GE Healthcare).

\section{Co-immunoprecipitation experiments}

Whole cell extracts diluted to $150 \mathrm{mM} \mathrm{NaCl}(1-2$ $\mathrm{mg}$ ) were incubated with normal rabbit serum for 30 minutes and subsequently with protein A-sepharose beads (GE Healthcare) for 1 hour at $4^{\circ} \mathrm{C}$. After centrifugation, beads were discarded and supernatants incubated for 2 hours with anti- $\beta \operatorname{TrCP}$ rabbit monoclonal antibody (Cell Signaling) or normal rabbit serum, followed by protein A-sepharose beads for 1 hour. Beads were washed and bound proteins were solubilized by the addition of SDSsample buffer heated at $95^{\circ} \mathrm{C}$ for 5 minutes.

\section{Tandem mass spectrometry (MS/MS)}

HA- $\beta \operatorname{TrCP}$ was transfected into Cos-7 cells. After $18 \mathrm{~h}$, cells were treated for $4 \mathrm{~h}$ with LLnL and HA- $\beta \operatorname{TrCP}$ immunoprecipitated from nuclear and cytosolic fractions with anti-HA (Covance) previously coupled to a resin from Pierce (AminoLink Plus Coupling Resin). Resins were washed three times in NP-40 lysis buffer and six times in ammonium bicarbonate $50 \mathrm{mM}$. Samples were analyzed by MS/MS using a LTQ mass spectrometer (Thermo Electron) as described previously [40].

\section{In vitro and in vivo ubiquitination assays}

The in vitro ubiquitination of CDK1 was performed in a volume of $10 \mu 1$ containing $50 \mathrm{mM}$ Tris- $\mathrm{HCl}(\mathrm{pH}$ 7.6), $5 \mathrm{mM} \mathrm{MgCl}_{2}, 0.6 \mathrm{mM}$ DTT, $2 \mathrm{mM}$ ATP, $2 \mu 1$ in vitro transcribed/translated unlabeled F-box protein, $1.5 \mathrm{ng} / \mu \mathrm{l}$ E1 (His ${ }_{6}$-ubiquitin activating enzyme, Boston Biochem), 10ng/ $\mu 1 \mathrm{His}_{6}-\mathrm{UbcH} 3$ (E2, Boston Biochem), 10ng/ $\mu 1 \mathrm{UbcH} 5 \mathrm{a}$ (E2, Boston Biochem), $2.5 \mu \mathrm{g} / \mu 1$ ubiquitin (Sigma), $1 \mu \mathrm{M}$ ubiquitin aldehyde (Boston Biochem), and $1 \mu 1{ }^{35} \mathrm{~S}$-methionine-labelled in vitro transcribed/translated $\mathrm{CDK} 1$ as substrate. The reactions were incubated at $30^{\circ} \mathrm{C}$ for $1 \mathrm{~h}$ and analyzed by SDS-PAGE and autoradiography.

The in vivo ubiquitination experiments were performed in Cos-7 cells transfected and treated with ConA 4 hours before harvesting. Cells were washed in PBS, lysed at $95^{\circ} \mathrm{C}$ for 15 minutes in NP40 buffer supplemented with $5 \%$ SDS and $10 \mathrm{mM}$ iodoacetamide, and diluted in NP40 buffer supplemented with $10 \mathrm{mM}$ iodoacetamide. Flag-CDK1 was immunoprecipitated and proteins separated by SDS-PAGE, electroblotted and probed with different antibodies.

\section{Immunofluorescence microscopy}

HeLa cells were grown on coverslips, treated or not with LLnL for 4 hours, and fixed with $4 \%$ paraformaldehyde and permeabilized with $0.1 \%$ Triton $\mathrm{X}-100$. Immunostaining, using monoclonal anti-CDK1 (Santa Cruz) and polyclonal anti-LC3 (Novus Biologicals) antibodies, and counterstaining with DAPI to visualize the nuclei, was carried out according to standard protocols. Epifluorescence microscopy was performed using a Leica microscope.

\section{Tumor immunohistochemistry}

Two tissue microarrays, one containing 66 human carcinomas of different sites (breast (6), prostate (5), liver (3), stomach (4), pancreato-biliary (6), colorectal (4), lung (10), head and neck (4), kidney (4), bladder (4), ovary (5), cervix (3), endometrium (2), and thyroid (6), and one with 109 carcinomas of the prostate, were used for immunohistochemistry. All the carcinoma tissues (1$\mathrm{mm}$ cores) were collected from archival paraffin blocks at this hospital, with the approval of the institutional ethical board. After dewaxing, sections were subjected to heatinduced epitope retrieval in 10mM EDTA pH 9.0, and were incubated with either anti- $\beta \operatorname{TrCP}$ rabbit polyclonal antibody (1:150; Cell Signaling) or anti-CDK1 rabbit polyclonal antibody (1:400; Santa Cruz) overnight at $4^{\circ} \mathrm{C}$. Peroxidase-labeled secondary reagents, chromogen (diaminobenzidine), and hematoxylin counterstain were applied according to manufacturer's protocols (Dako). Immunostaining was evaluated by two observers and scored as low $(<10 \%)$, moderate $(10-25 \%)$, or high $(>25 \%)$, according to the percentage of immunostained cells. Moderate and high categories were combined as positive for the purpose of statistical analysis. Prostate tumors were classified into low grade or high grade according to Gleason score, the latter being tumors with Gleason score $4+3$ or higher. Statistical analyses were 
performed on contingency tables using Fisher's exact test. Two-sided $\mathrm{p}$ value $<0.05$ was considered as significant.

\section{ACKNOWLEDGEMENTS}

This work was supported by Spanish grants from Ministerio de Economía y Competitividad, MINECO [SAF2011-30003], Instituto de Salud Carlos III [PI13/02282] and Dirección General de Investigación, Tecnología y Empresa, Junta de Andalucía, DGITE [P08CVI-03603 and P10-CTS-6243]. MM-S was supported by a fellowship from MINECO, SG by DGITE and CS by Programa Nicolás Monardes.

The authors declare that they have no conflict of interest.

\section{REFERENCES}

1. Lim S and Kaldis P. Cdks, cyclins and CKIs: roles beyond cell cycle regulation. Development. 2013; 140(15):30793093 .

2. Aleem E, Kiyokawa $\mathrm{H}$ and Kaldis P. Cdc2-cyclin E complexes regulate the $\mathrm{G} 1 / \mathrm{S}$ phase transition. Nature cell biology. 2005; 7(8):831-836.

3. Santamaria D, Barriere C, Cerqueira A, Hunt S, Tardy C, Newton K, Caceres JF, Dubus P, Malumbres M and Barbacid M. Cdk1 is sufficient to drive the mammalian cell cycle. Nature. 2007; 448(7155):811-815.

4. Gopinathan L, Ratnacaram CK and Kaldis P. Established and novel $\mathrm{Cdk} /$ cyclin complexes regulating the cell cycle and development. Results and problems in cell differentiation. 2011; 53:365-389.

5. Smits VA and Medema RH. Checking out the $G(2) / M$ transition. Biochimica et biophysica acta. 2001; 1519(12):1-12.

6. Takizawa CG and Morgan DO. Control of mitosis by changes in the subcellular location of cyclin-B1-Cdk1 and Cdc25C. Current opinion in cell biology. 2000; 12(6):658665.

7. Donzelli $\mathrm{M}$ and Draetta GF. Regulating mammalian checkpoints through Cdc25 inactivation. EMBO reports. 2003; 4(7):671-677.

8. Featherstone $\mathrm{C}$ and Russell P. Fission yeast p107wee1 mitotic inhibitor is a tyrosine/serine kinase. Nature. 1991; 349(6312):808-811.

9. Mueller PR, Coleman TR, Kumagai A and Dunphy WG. Myt1: a membrane-associated inhibitory kinase that phosphorylates Cdc 2 on both threonine-14 and tyrosine- 15 . Science. 1995; 270(5233):86-90.

10. Morgan DO. (2007). The cell cycle: principles of control. (London: New Science Press Ltd.)

11. Welch PJ and Wang JY. Coordinated synthesis and degradation of cdc2 in the mammalian cell cycle. Proceedings of the National Academy of Sciences of the
United States of America. 1992; 89(7):3093-3097.

12. Yoon $\mathrm{CH}$, Miah MA, Kim KP and Bae YS. New Cdc2 Tyr 4 phosphorylation by dsRNA-activated protein kinase triggers Cdc2 polyubiquitination and G2 arrest under genotoxic stresses. EMBO reports. 2010; 11(5):393-399.

13. Xu P, Duong DM, Seyfried NT, Cheng D, Xie Y, Robert J, Rush J, Hochstrasser M, Finley D and Peng J. Quantitative proteomics reveals the function of unconventional ubiquitin chains in proteasomal degradation. Cell. 2009; 137(1):133145.

14. Schreiber A and Peter M. Substrate recognition in selective autophagy and the ubiquitin-proteasome system. Biochimica et biophysica acta. 2014; 1843(1):163-181.

15. Gavilan E, Sanchez-Aguayo I, Daza P and Ruano D. GSK3beta signaling determines autophagy activation in the breast tumor cell line MCF7 and inclusion formation in the non-tumor cell line MCF10A in response to proteasome inhibition. Cell death \& disease. 2013; 4:e572.

16. Limon-Mortes MC, Mora-Santos M, Espina A, Pintor-Toro JA, Lopez-Roman A, Tortolero M and Romero F. UVinduced degradation of securin is mediated by SKP1-CUL1beta TrCP E3 ubiquitin ligase. Journal of cell science. 2008; 121(Pt 11):1825-1831.

17. Fuchs SY, Spiegelman VS and Kumar KG. The many faces of beta-TrCP E3 ubiquitin ligases: reflections in the magic mirror of cancer. Oncogene. 2004; 23(11):2028-2036.

18. Siu WY, Yam CH and Poon RY. G1 versus G2 cell cycle arrest after adriamycin-induced damage in mouse Swiss3T3 cells. FEBS letters. 1999; 461(3):299-305.

19. Golsteyn RM. Cdk1 and Cdk2 complexes (cyclin dependent kinases) in apoptosis: a role beyond the cell cycle. Cancer letters. 2005; 217(2):129-138.

20. Reinhardt HC and Yaffe MB. Phospho-Ser/Thr-binding domains: navigating the cell cycle and DNA damage response. Nature reviews Molecular cell biology. 2013; 14(9):563-580.

21. Mailand N, Podtelejnikov AV, Groth A, Mann M, Bartek $\mathrm{J}$ and Lukas J. Regulation of G(2)/M events by Cdc25A through phosphorylation-dependent modulation of its stability. The EMBO journal. 2002; 21(21):5911-5920.

22. Badie C, Itzhaki JE, Sullivan MJ, Carpenter AJ and Porter AC. Repression of CDK1 and other genes with CDE and CHR promoter elements during DNA damage-induced $\mathrm{G}(2) / \mathrm{M}$ arrest in human cells. Molecular and cellular biology. 2000; 20(7):2358-2366.

23. Cheng JD, Dunbrack RL, Jr., Valianou M, Rogatko A, Alpaugh RK and Weiner LM. Promotion of tumor growth by murine fibroblast activation protein, a serine protease, in an animal model. Cancer research. 2002; 62(16):4767-4772.

24. Seki Y, Suico MA, Uto A, Hisatsune A, Shuto T, Isohama $\mathrm{Y}$ and Kai $\mathrm{H}$. The ETS transcription factor MEF is a candidate tumor suppressor gene on the $\mathrm{X}$ chromosome. Cancer research. 2002; 62(22):6579-6586.

25. Tremblay L, Hauck W, Aprikian AG, Begin LR, 
Chapdelaine A and Chevalier S. Focal adhesion kinase (pp125FAK) expression, activation and association with paxillin and $550 \mathrm{CSK}$ in human metastatic prostate carcinoma. International journal of cancer Journal international du cancer. 1996; 68(2):164-171.

26. Suzuki H, Romano-Spica V, Papas TS and Bhat NK. ETS1 suppresses tumorigenicity of human colon cancer cells. Proceedings of the National Academy of Sciences of the United States of America. 1995; 92(10):4442-4446.

27. Wu Y, Pan S, Luo W, Lin SH and Kuang J. Hp95 promotes anoikis and inhibits tumorigenicity of HeLa cells. Oncogene. 2002; 21(44):6801-6808.

28. Yoon CH, Lee ES, Lim DS and Bae YS. PKR, a p53 target gene, plays a crucial role in the tumor-suppressor function of p53. Proceedings of the National Academy of Sciences of the United States of America. 2009; 106(19):7852-7857.

29. Klotz K, Cepeda D, Tan Y, Sun D, Sangfelt O and Spruck C. $\mathrm{SCF}(\mathrm{Fbxw} 7 / \mathrm{hCdc} 4)$ targets cyclin E2 for ubiquitindependent proteolysis. Experimental cell research. 2009; 315(11):1832-1839.

30. Margottin F, Bour SP, Durand H, Selig L, Benichou S, Richard V, Thomas D, Strebel K and Benarous R. A novel human WD protein, h-beta TrCp, that interacts with HIV-1 Vpu connects CD4 to the ER degradation pathway through an F-box motif. Mol Cell. 1998; 1(4):565-574.

31. Fukuda T, Naiki T, Saito M and Irie K. hnRNP K interacts with RNA binding motif protein 42 and functions in the maintenance of cellular ATP level during stress conditions. Genes to cells : devoted to molecular \& cellular mechanisms. 2009; 14(2):113-128.

32. Bunz F, Dutriaux A, Lengauer C, Waldman T, Zhou S, Brown JP, Sedivy JM, Kinzler KW and Vogelstein B. Requirement for p53 and p21 to sustain G2 arrest after DNA damage. Science. 1998; 282(5393):1497-1501.

33. Romero F, Gil-Bernabe AM, Saez C, Japon MA, PintorToro JA and Tortolero M. Securin is a target of the UV response pathway in mammalian cells. Molecular and cellular biology. 2004; 24(7):2720-2733.

34. Jin DY, Spencer F and Jeang KT. Human T cell leukemia virus type 1 oncoprotein Tax targets the human mitotic checkpoint protein MAD1. Cell. 1998; 93(1):81-91.

35. Romero F, Germani A, Puvion E, Camonis J, VarinBlank N, Gisselbrecht S and Fischer S. Vav binding to heterogeneous nuclear ribonucleoprotein (hnRNP) C. Evidence for Vav-hnRNP interactions in an RNAdependent manner. The Journal of biological chemistry. 1998; 273(10):5923-5931.

36. Gil-Bernabe AM, Romero F, Limon-Mortes MC and Tortolero M. Protein phosphatase 2A stabilizes human securin, whose phosphorylated forms are degraded via the SCF ubiquitin ligase. Molecular and cellular biology. 2006; 26(11):4017-4027.

37. Arnaud N, Dabo S, Maillard P, Budkowska A, Kalliampakou KI, Mavromara P, Garcin D, Hugon J,
Gatignol A, Akazawa D, Wakita T and Meurs EF. Hepatitis $\mathrm{C}$ virus controls interferon production through PKR activation. PloS one. 2010; 5(5):e10575.

38. Lee JY, Yu SJ, Park YG, Kim J and Sohn J. Glycogen synthase kinase 3beta phosphorylates p21WAF1/CIP1 for proteasomal degradation after UV irradiation. Molecular and cellular biology. 2007; 27(8):3187-3198.

39. Mailand N, Bekker-Jensen S, Bartek J and Lukas J. Destruction of Claspin by SCFbetaTrCP restrains Chk1 activation and facilitates recovery from genotoxic stress. Mol Cell. 2006; 23(3):307-318.

40. Barquilla A, Saldivia M, Diaz R, Bart JM, Vidal I, Calvo E, Hall MN and Navarro M. Third target of rapamycin complex negatively regulates development of quiescence in Trypanosoma brucei. Proceedings of the National Academy of Sciences of the United States of America. 2012; 109(36):14399-14404. 